\title{
Modeling of a Piezoelectric MEMS Micropump Dedicated to Insulin Delivery and Experimental Validation Using Integrated Pressure Sensors: Application to Partial Occlusion Management
}

\author{
S. Fournier and E. Chappel \\ Debiotech S.A., 28 avenue de Sévelin, 1004 Lausanne, Switzerland \\ Correspondence should be addressed to E. Chappel; e.chappel@debiotech.com
}

Received 1 March 2017; Accepted 10 May 2017; Published 31 May 2017

Academic Editor: Paolo Bruschi

Copyright (C) 2017 S. Fournier and E. Chappel. This is an open access article distributed under the Creative Commons Attribution License, which permits unrestricted use, distribution, and reproduction in any medium, provided the original work is properly cited.

\begin{abstract}
A numerical model based on equivalent electrical networks has been built to simulate the dynamic behavior of a positivedisplacement MEMS micropump dedicated to insulin delivery. This device comprises a reservoir in direct communication with the inlet check valve, a pumping membrane actuated by a piezo actuator, two integrated piezoresistive pressure sensors, an antifree-flow check valve at the outlet, and finally a fluidic pathway up to the patient cannula. The pressure profiles delivered by the sensors are continuously analyzed during the therapy in order to detect failures like occlusion. The numerical modeling is a reliable way to better understand the behavior of the micropump in case of failure. The experimental pressure profiles measured during the actuation phase have been used to validate the numerical modeling. The effect of partial occlusion on the pressure profiles has been also simulated. Based on this analysis, a new management of partial occlusion for MEMS micropump is finally proposed.
\end{abstract}

\section{Introduction}

As microsystems are becoming more complex, simulation and modeling become more relevant and widespread in the micropump domain. Zengerle and Richter have shown in 1994 that micropumps could be modeled using the equivalence between fluidic systems and electrical networks [1]. Bourouina et al. have verified and used this method to design a micropump for medical application $[2,3]$. More recently, thanks to progress in the computational domain, models can be driven using software such as ANSYS for a complete electro-fluid-solid simulation [4]. Experimental validation of the modeling of a piezoelectric micropump was already reported for a device with no-moving-part valves [5] and a valveless [6] and piezoelectric peristaltic micropump [7].

This paper presents a piezoelectric positive-displacement MEMS micropump having two check valves and a fixed stroke volume $[8,9]$. The main features of this device are briefly described in Section 2. The modeling of the fluid dynamics inside the device using electrical equivalent networks is provided in Section 3. The experimental validation of this modeling, based on the analysis of the pressure profiles provided by integrated pressure sensors, is finally proposed in Section 4 with a specific discussion about partial occlusion management.

\section{Design and Working Principle}

A piezoelectric positive-displacement MEMS micropump is considered here. Figure 1(a) shows a cross section of the pump chip, made of a stack of three plates: top and bottom caps in silicon bonded to a SOI wafer in between. Overall chip dimensions are $10 \mathrm{~mm} \times 6 \mathrm{~mm} \times 1.4 \mathrm{~mm}$ (length $\times$ width $\times$ height). The direction of the flow is indicated by arrows. The fluidic pathway comprises (from left to right) the following: the inlet port in direct communication with the reservoir filter; the inlet valve; the pumping chamber; the inner pressure sensor; the outlet valve; the top channel; the 


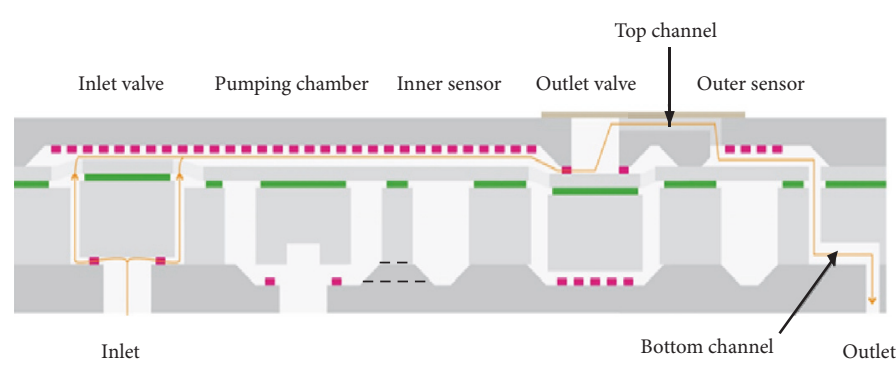

(a)

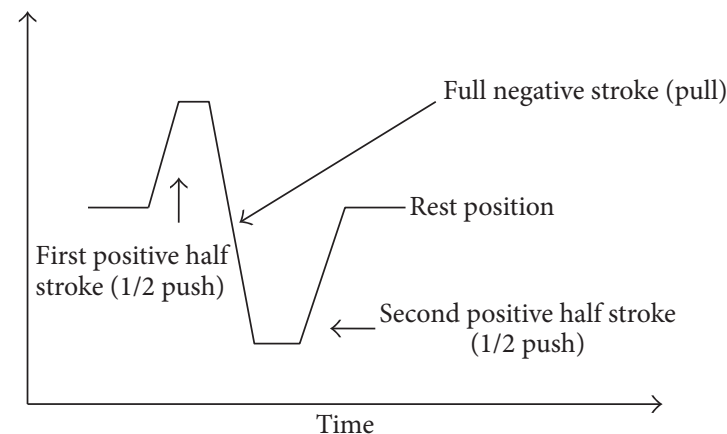

(b)

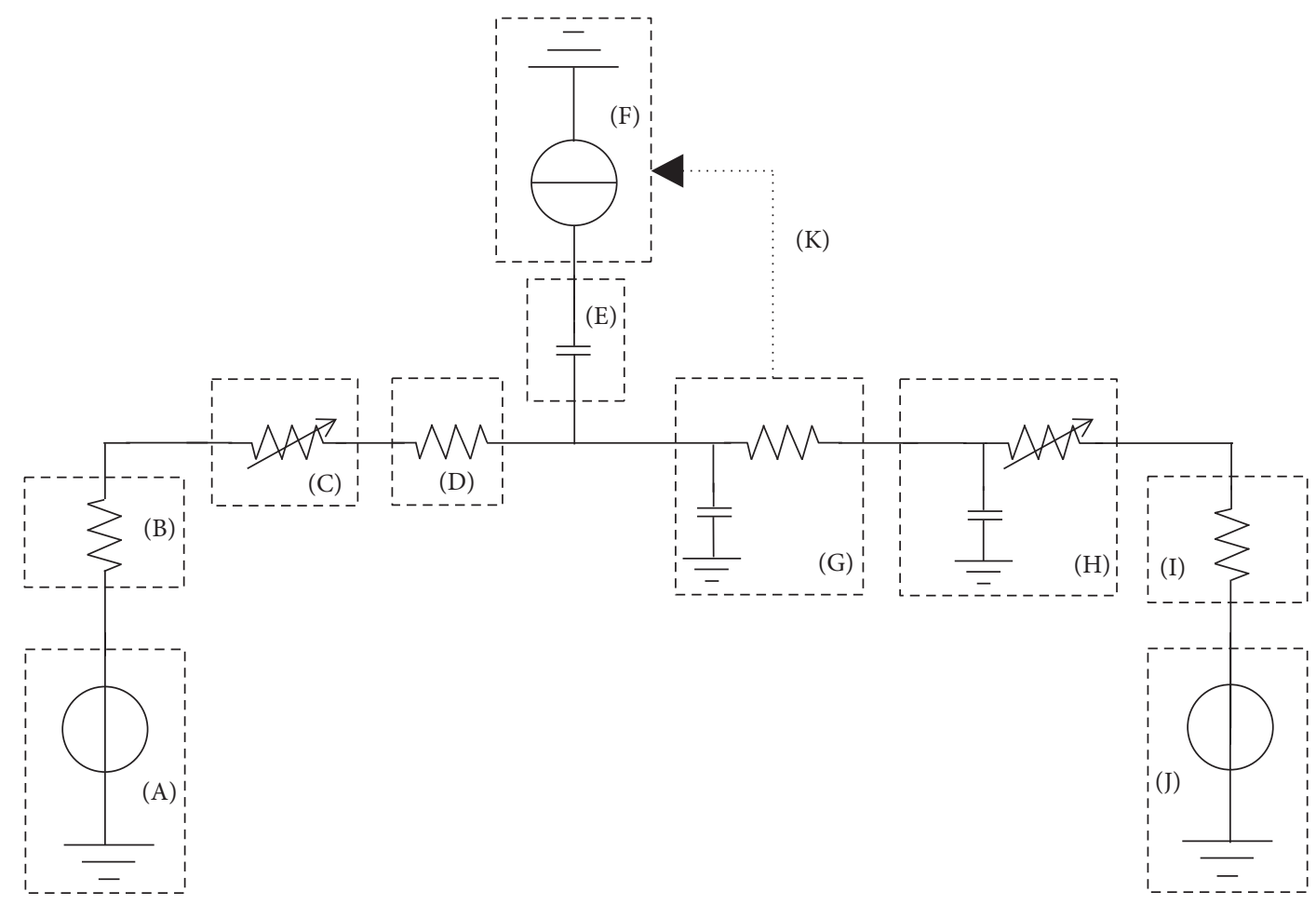

(c)

Figure 1: (a) Schematic cross section of the pump chip (not to scale). The mechanical stops (pink pads) present on both top and bottom wafers are also used as antistiction features. The buried oxide is represented by the thick green line under the device layer. The vent of the outer pressure sensor and the piezo actuator are not represented here. The top channel is covered by a glass cap. (b) Actuation profile applied on the piezoelectric bender. Positive part ("PUSH") corresponds to an upward movement of the pumping membrane and therefore to insulin infusion in the patient; negative part ("PULL") corresponds to a downward movement of the pumping membrane, leading to the filling of the chamber with insulin coming from the reservoir. (c) General electrical network modeling the different pump elements: the insulin reservoir (A), the particle filter (B), the inlet valve (C), the channel from the inlet valve to the pumping chamber (D), the pumping membrane (E), the actuation and the pumping membrane displacement $(\mathrm{F})$, the inner sensor $(\mathrm{G})$, the outlet valve $(\mathrm{H})$, the outer sensor $(\mathrm{I})$, the backpressure $(\mathrm{J})$, and the retroaction of the pressure in the pumping chamber on the membrane displacement $(\mathrm{K})$.

outer pressure sensor; the bottom channel; and finally the outlet, leading to the cannula port.

During the filling, while the membrane is pulled down, the outlet valve remains closed and the inlet valve opens when the underpressure in the pumping chamber reaches the inlet valve opening threshold. During the infusion, the actuator pushes the membrane against the upper mechanical stop, inducing an overpressure that opens the outlet valve and keeps the inlet valve closed (Figure 1(b)). The relative pressure inside the pumping chamber varies typically between
-800 mbar and +800 mbar during an actuation cycle. The stroke volume is about $200 \mathrm{~nL}$ and the compression ratio larger than 1.8 ensures self-priming capability. The operation of the pump is monitored by the two integrated pressure sensors made of strain gauges implanted into silicon membranes: a first sensor is located inside the pumping chamber (hereafter the inner sensor), and a second sensor is located downstream of the outlet valve (hereafter the outer sensor) in order to monitor the patient pressure. Both sensor membranes are squares of about $1 \mathrm{~mm}^{2}$, with a thickness of $15 \mu \mathrm{m}$. 
TABLE 1: Physical and/or fluidic effects and their electrical equivalents.

\begin{tabular}{lc}
\hline Fluidic parameters & Electrical parameters \\
\hline Flow rate & Current \\
Pressure drop & Voltage \\
Mass & Inductance \\
Flow restrictor & Resistance \\
Elasticity & Capacitance \\
\hline
\end{tabular}

The physical characteristics of these gauge pressure sensors have been optimized to get a maximum offset of $\pm 2 \mathrm{mV} \cdot \mathrm{V}^{-1} \cdot$ bar $^{-1}$ and a sensitivity of $18 \mathrm{mV} \cdot \mathrm{V}^{-1} \cdot \mathrm{bar}^{-1}$ in the range -1 to +1.5 bar. The minimum resolution is $1 \mathrm{mbar}$. The micropump numerical modeling will provide simulated pressure profiles measured by both sensors during actuation.

\section{Methods}

The pumping system is divided into simple lumped elements. Regarding physical equations governing flow rate and pressure in a fluidic pathway, an equivalence with physical equations governing, respectively, current and voltage in an electrical network can be established. As a consequence of this equivalence between mechanical impedances (pressure drop to flow rate ratio) and electrical impedances (voltage to current ratio) [2], the following standard analogy between physical systems and electrical networks is considered as shown in Table 1.

In first approximation, regarding the microscopic scale of the system, the inertial effects have been neglected since the maximum Reynolds number does not exceed three hundred. The flow is therefore considered as laminar. If not explicitly specified, the temperature is $20^{\circ} \mathrm{C}$ for both simulations and experimental tests.

3.1. Microchannels. All the microchannels of the micropump are, in first approximation, considered as plane, rigid (purely resistive), with a width $a$, a depth $b$, and a length $L$ (with $b \ll a$ and $b \ll L$ ). The dynamic viscosity of the fluid is noted $\eta$. Therefore, the Navier-Stokes equations lead to the plane Poiseuille flow, which gives the pressure drop to flow rate ratio (1), classically named the hydraulic resistance:

$$
R_{f}=\frac{\Delta P}{\Phi}=\frac{12 \eta L}{a b^{3}}
$$

Microchannels are modeled as electrical resistance (see Table 2 for the numerical values).

3.2. Elasticity of Structural Elements. Zengerle and Richter have shown that for incompressible fluids elastic elements of a micropump are characterized by their pressure dependent volume deformation, called the fluid capacitance, and defined in formula (2):

$$
C=\rho \frac{d V}{d P}
$$

TABLE 2: Numerical values of the different parameters used to

\begin{tabular}{|c|c|c|}
\hline $\begin{array}{l}\text { Network } \\
\text { element }\end{array}$ & Description & Value \\
\hline (a) & Reservoir pressure & $0 \mathrm{~Pa}$ \\
\hline (b) & Particle filter resistance & $0 \mathrm{~Pa} \cdot \mathrm{s} / \mathrm{m}^{3}$ \\
\hline (c) & Inlet valve stiffness & $1.05 \mathrm{kN} / \mathrm{m}$ \\
\hline (d) & $\begin{array}{l}\text { Fluidic resistance between inlet } \\
\text { valve and pumping chamber }\end{array}$ & $2 \cdot 10^{12} \mathrm{~Pa} \cdot \mathrm{s} / \mathrm{m}^{3}$ \\
\hline (e) & $\begin{array}{l}\text { Pumping membrane compliance } \\
\mathrm{dV} / \mathrm{dP}\end{array}$ & $0.0043 \mathrm{nl} / \mathrm{mbar}$ \\
\hline \multirow{3}{*}{ (f) } & Piezoelectric actuator force factor & $0.082 \mathrm{~N} / \mathrm{V}$ \\
\hline & Max piezo voltage & $200 \mathrm{~V}$ \\
\hline & Membrane stiffness & $63 \mathrm{kN} / \mathrm{m}$ \\
\hline \multirow[b]{2}{*}{ (g) } & Inner sensor compliance $\mathrm{dV} / \mathrm{dP}$ & $0.00302 \mathrm{nl} / \mathrm{mbar}$ \\
\hline & $\begin{array}{l}\text { Fluidic resistance of inner } \\
\text { detector }\end{array}$ & $5 \cdot 10^{12} \mathrm{~Pa} \cdot \mathrm{s} / \mathrm{m}^{3}$ \\
\hline \multirow{2}{*}{ (h) } & Outlet valve stiffness & $20.8 \mathrm{kN} / \mathrm{m}$ \\
\hline & Outer valve compliance $\mathrm{dV} / \mathrm{dP}$ & $0.00256 \mathrm{nl} / \mathrm{mbar}$ \\
\hline (i) & $\begin{array}{l}\text { Fluidic resistance of outer } \\
\text { detector }\end{array}$ & $6.7 \cdot 10^{12} \mathrm{~Pa} \cdot \mathrm{s} / \mathrm{m}^{3}$ \\
\hline (j) & Backpressure & $0 \mathrm{~Pa}$ \\
\hline
\end{tabular}
validate the model as shown in Figures 2 and 3.

with $\rho$ being the fluid density [1]. Therefore, elasticity is modeled as an electrical capacitance.

Structural elements exhibit finite stiffness and can slightly change the volume inside the chamber during the pump operation. FEM analysis shows that three flexible elements have a nonnegligible contribution to the stroke volume, depending on the pressure: the inner sensor, the outlet valve, and the pumping membrane (at full deflection against mechanical stops). Their typical variations of volume per unit of pressure are given in Table 2. The compressibility of water is equal to $\chi=5 \cdot 10^{-5} \mathrm{bar}^{-1}$. Considering a total dead volume of $249 \mathrm{~nL}$ at full membrane deflection, the volume change of this dead volume per unit of pressure is only 1.2 . $10^{-5} \mathrm{~nL} / \mathrm{mbar}$. Therefore it is assumed in the model that water is incompressible.

3.3. Pressure Sensors. Considering the analogy provided in Table 1, the two pressure sensors are modeled as voltmeters.

3.4. Check Valves. Inlet and outlet valves are passive check valves consisting in two circular pillars, lying on valve seats. The Navier-Stokes equations for such radial flow between two parallel disks (and forming a fluidic ring) lead to the expression (3) for the variable hydraulic resistance of the valve [10]:

$$
R_{f \text { Valve }}=\frac{6 \eta}{\pi h^{3}} \log \left(\frac{r_{d}}{r_{u}}\right)
$$

where $r_{u}$ is the radius of the inlet hole (or inner radius of the fluidic ring), $r_{d}$ the outlet hole (or outer radius of the fluidic 
ring), and $h$ the valve opening (or thickness of the fluidic ring), given by

$$
h=\frac{\Delta P \cdot S}{k_{\text {Valve }}},
$$

where $\Delta P$ is the pressure drop across the valve, $S$ the surface of the inlet hole, and $k_{\text {Valve }}$ the valve stiffness determined by means of FEM simulations. The valve stiffness is induced by the arms (for the inlet valve) or the membrane (outlet valve) linking the pillars to the SOI wafer. The valve is modeled as a variable electrical resistance, and its opening is calculated by measuring the forward and reverse pressures with voltmeters.

3.5. Actuation Elements. The steady state approximation is applied to write the equilibrium between the different forces acting on the pumping membrane: the actuation force $F_{B}$, proportional to the voltage $U(t)$ applied on the piezoelectric bender; the counterpressure force $F_{P}$, due to the fluid inside the pumping chamber; the elastic restoring force of the membrane $F_{E}$, coupled with the piezoelectric bender and the mesa; and the mechanical stop forces $F_{\mathrm{Up}}$ and $F_{\text {Down }}$, acting as linear springs only when the membrane reaches the upper or lower mechanical stops. Equilibrium equation (5), providing the membrane displacement, writes

$$
F_{B}=F_{P}+F_{E}+F_{\mathrm{Up}}+F_{\text {Down }} .
$$

The flow rate $\Phi(t)$ is derived from the membrane displacement $\delta(t)$ and controls the dynamics of the system (especially $P_{\text {chamber }}(t)$ ) as schematized by

$$
U(t) \longrightarrow \underset{\hat{i}}{\delta}(t) \longrightarrow \Phi(t) \longrightarrow P_{\text {chamber }}(t) .
$$

In the simulation, the flow rate generated by the displacement is modeled as a controlled current source (Figure 1(c), element (F)).

3.6. General Network. Previous parts described the different elementary blocks needed for building the general network (Figure 1(c)). Two controlled voltage sources are added at each extremity of the fluidic network to model (i) the pressure inside the reservoir and (ii) the backpressure in the patient cannula. The capacitances modeling the compliance of the structural elements are placed in parallel with other components. In standard operation the fluid flows from left to right (Figure 1(c)).

3.7. Modeling a Cannula Occlusion. Mechanical occlusions may occur during the pump lifetime. Typical root causes are as follows: cannula bending; accumulation, fibrillation, or drying of insulin; or a flow restriction in the cannula due to the subcutaneous tissue. To simulate such phenomenon, we added an equivalent electrical resistance at the end of the network.

To model experimentally such a restriction, circular microtubes were added at the outlet port (internal diameter ID $=90 \mu \mathrm{m}$ ) with a total length $L_{\text {occlusion }}$ (the longer the tube, the bigger the occlusion). According to the HagenPoiseuille law [11], derived from the application of NavierStokes equations in a cylindrical tube, the hydraulic resistance of this terminal tube writes

$$
R_{\text {occlusion }}=\frac{128 \eta L_{\text {occlusion }}}{\pi d^{4}} .
$$

The cannula elasticity was modeled by adding a capacitance in parallel.

\section{Results}

The effects of leakage and overfilling have been already studied experimentally [9]. A focus is therefore made on occlusion, one of the major failure modes in CSII (continuous subcutaneous insulin infusion). Occlusion rate is notably increased by temperature due to insulin fibrillation [12] and by late catheter change that can lead to insulin crystallization [13]. The numerical model has been used to optimize the actuation profile in order to meet accuracy requirement in case of partial occlusion.

For our simulations, the insulin is considered as equivalent to water in terms of compressibility, viscosity, and density. Valve pretensions have been fixed at $80 \mathrm{mbar}$ for the inlet and $70 \mathrm{mbar}$ for the outlet. The experimental setup used to validate the model is described in [9]. PEEK microtubes and fittings used to simulate partial occlusions are manufactured by Upchurch Scientific.

4.1. Model Validation in Standard Operation. For the inner sensor, a first positive peak of pressure starting at $t=$ $0.1 \mathrm{~s}$ is observed during the first positive half stroke of the piezoelectric bender (1/2 push) as described in Figure 1(b). Insulin is infused towards the patient if the pressure inside the pumping chamber is larger than the outlet valve pretension. The piezoelectric bender is maintained at high voltage during $0.3 \mathrm{~s}$ and at the time $t=0.43 \mathrm{~s}$ a full negative stroke is performed to fill the pumping chamber with insulin coming from the reservoir. During this phase a large negative peak of pressure is monitored, followed by another stabilization period of $0.3 \mathrm{~s}$. Finally a positive peak of pressure at $t=0.8 \mathrm{~s}$ is observed during the second positive half stroke of the bender (see Figure 1(b)).

The simulated pressure profiles match the experimental data (see Figure 2). The simulation underestimates the positive pressure peak only by $4.4 \%$ and overestimates the negative pressure peak only by $1.1 \%$.

For the outer sensor, two pressure peaks are visible, corresponding to the positive peaks of the inner sensor and bringing out the moments and durations of the infusion of insulin in the patient. The first pressure peak is overestimated by $3.2 \%$ by the numerical model.

The slight overestimations of the pressure values can be explained by the fact that the time step used in the experimental data is 10 to 100 times larger than the one used in the simulated data.

Finally, the simulated infused volume is $V_{\text {Model }}=209.8 \mathrm{~nL}$ which is overestimated by $1.6 \%$ compared to the nominal stroke volume. 


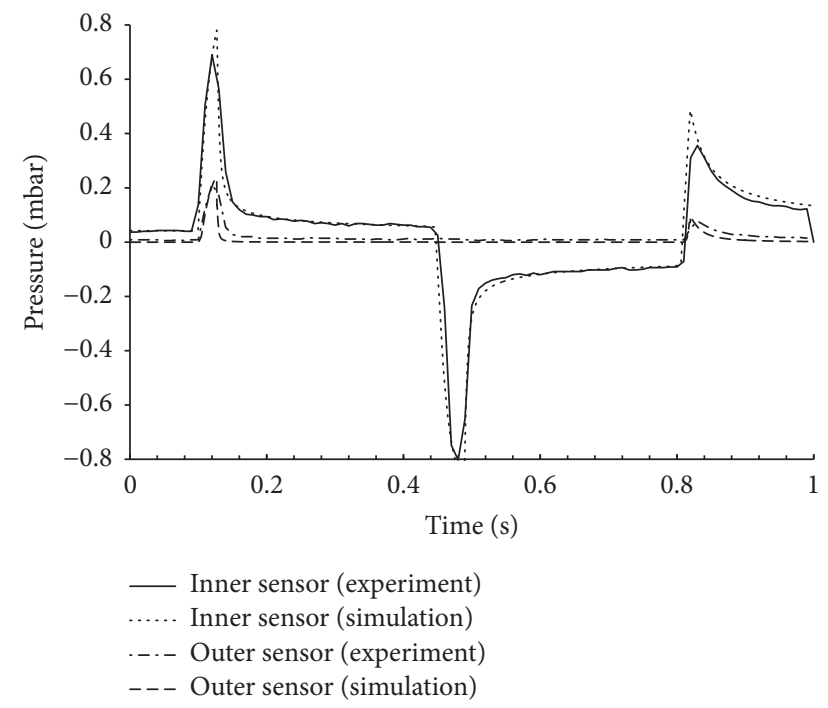

Figure 2: Comparisons between experimental and simulated pressure profiles for the inner and outer sensors during the normal operation of the pump (no occlusion).

4.2. Model Validation in Case of Partial Occlusion. Partial occlusion of the cannula has been simulated using $L_{\text {occlusion }}$ $=20 \mathrm{~cm}$. The simulation results show again good agreement with experimental data (see Figure 3). For the inner sensor, the combination of the "PUSH" actuation and the flow restrictor generates higher levels of pressure for the positive peaks and a slower pressure decay while the piezoelectric bender is held in the "PUSH" position: the infusion of insulin is more difficult and slower. The value of the positive peak is slightly overestimated by $6 \%$ by the numerical model. The negative part of the signal is not affected by the occlusion. For the outer sensor, the two pressure peaks are both affected as well as the two positive pressure peaks of the inner sensor. The value of the first positive peak is overestimated by $40 \%$ which is more than the difference observed for the inner sensor. This error is attributed to the very rough estimation of the fluidic pathway compliance downstream of the micropump.

4.3. Actuation Cycle and Partial Occlusion. The temperature is now set at $37^{\circ} \mathrm{C}$ with a fluid viscosity of $0.69 \mathrm{cP}$. The simulations of the pressure profiles as a function of $L_{\text {occlusion }}$ are shown for both pressure sensors (see Figure 4 for the inner sensor and Figure 5 for the outer sensor). According to Figure 1(a), the outlet valve is closed when the pressure in the pumping chamber is, in theory, lower than the outlet valve pretension. In practice, since the fluidic resistance of the valve varies as the power -3 of the valve opening $h$ (see (3)), the outlet valve can be considered as substantially closed when the pressure reaches a threshold value $P_{\text {th }}$ that depends on valve geometry.

It shall be noted that accuracy is notably conditioned by the absence of backflow through the outlet valve during actuation: the outlet valve shall be closed when the pump filling phase is initialized. This is achieved when the pressure in the pumping chamber at the end of the infusion cycle is

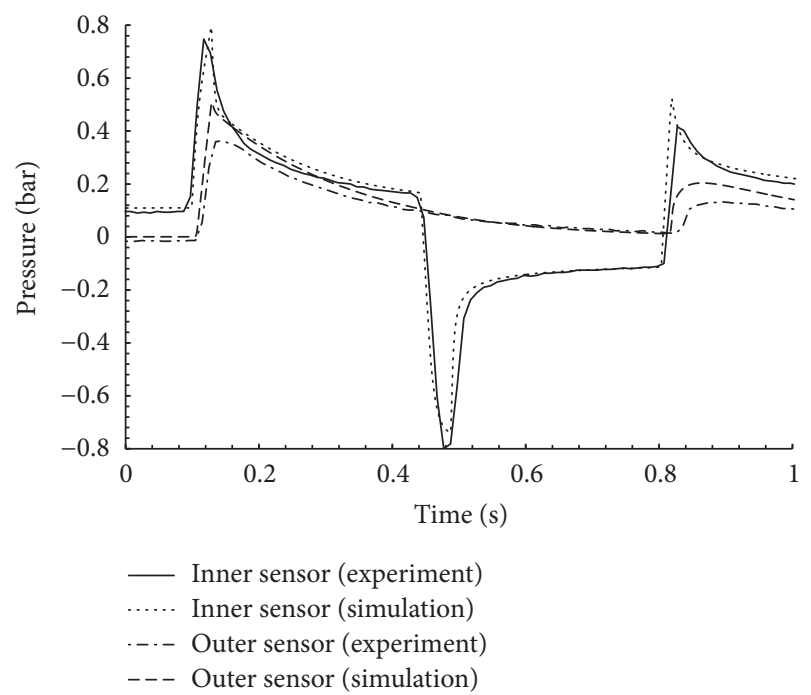

FIGURE 3: Comparisons between experimental and simulated pressure profiles for the inner and outer sensors during the first actuation cycle following a partial occlusion $\left(L_{\text {occlusion }}=20 \mathrm{~cm}\right)$.

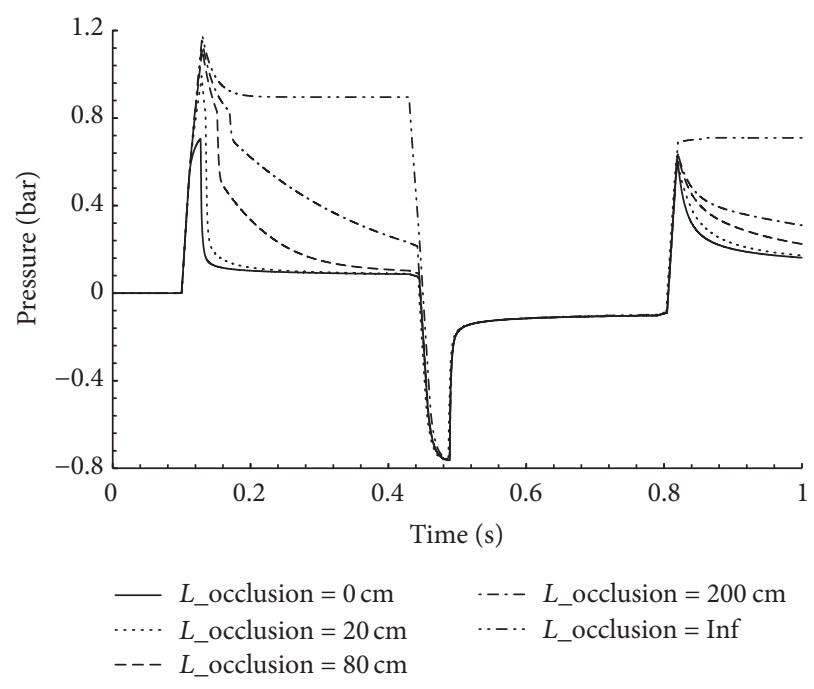

FIGURE 4: Simulated pressure profiles of the inner sensor as a function of the occlusion length defined in (7).

equal to or lower than $P_{\text {th }}$. Considering an outlet pretension of 70 mbar, simulation data show that a value of $P_{\text {th }} \approx 200 \mathrm{mbar}$ is compatible with accuracy requirement of $\pm 5 \%$. The presence of a partial occlusion increases the time necessary to reach this threshold value as shown in Figure 4.

During the infusion phase, the piezo actuator is powered during a period called "hold time" in order to maintain the pumping membrane against the mechanical stops. This hold time shall be therefore adjusted in case of partial occlusion in order to infuse the correct amount of insulin. This feature is particularly interesting during overnight basal infusion to reduce the occurrence of occlusion alarm (due to, for instance, tissue compression if the patient sleeps onto its patch pump) and to limit the risk of cannula leakage (induced by the large generated pressure at the outlet, see Figure 5). 
TABLE 3: Simulated values of the piezo actuator hold time in ms as a function of the occlusion length in $\mathrm{cm}$ and the outlet valve pretension in mbar.

\begin{tabular}{lccccc}
\hline \multirow{2}{*}{ Pretension (mbar) } & \multicolumn{5}{c}{ Occlusion length $(\mathrm{cm})$} \\
& 30 & 50 & 70 & 120 & 200 \\
\hline 80 & 320 & 510 & 710 & 1200 & 1990 \\
100 & 330 & 530 & 730 & 1230 & 2050 \\
120 & 340 & 550 & 750 & 1270 & 2110 \\
\hline
\end{tabular}

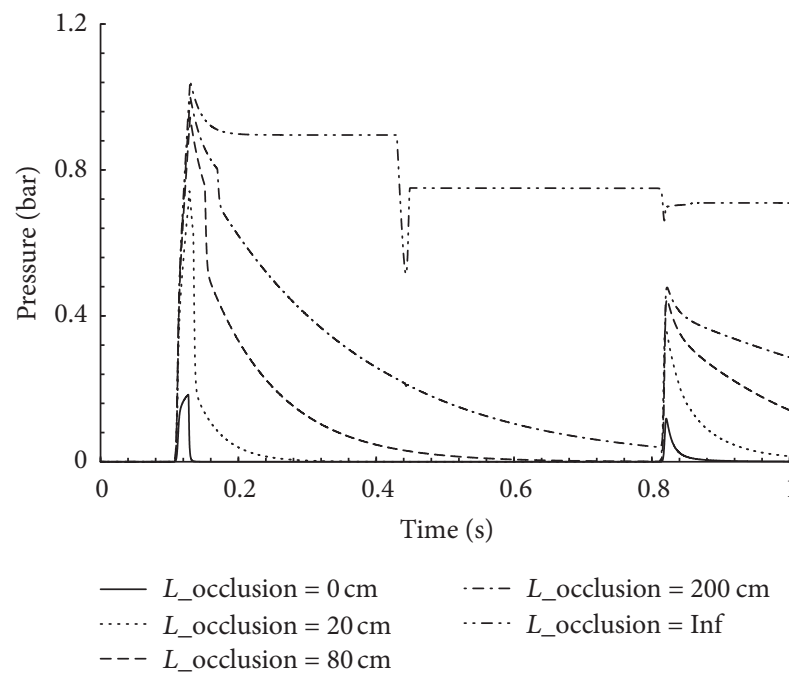

FIGURE 5: Simulated pressure profiles of the outer sensor as a function of the occlusion length defined in (7).

Typical hold times as a function of outlet valve pretensions and occlusion lengths are provided in Table 3 . These values are compatible with all basal infusion rates (up to 10 units of insulin U100 per hour).

To better visualize the correlation between partial occlusion value and delivery accuracy, the opening of the outlet valve during the actuation cycle is provided in Figure 6. A minimum opening of 0.1 micron has been introduced in the model to prevent singularities. The outlet valve is fully open during total occlusion as expected. In case of partial occlusion, the time to close the valve is directly impacted by the increase of the downstream fluidic restriction. The valve opening shall be smaller than about 1 micron before actuating the piezo actuator for the pump filling phase.

Figure 7 shows the simulated pumping membrane displacements during an actuation cycle, for various occlusion lengths. The time to get a full membrane displacement is significantly affected by the value of $L_{\text {occlusion. }}$. In case of total occlusion the pumping membrane is never in contact with the upper mechanical stops due to the large generated pressure and the finite piezo blocking force. During the final part of the actuation cycle, when the piezo voltage is set to zero, the pumping membrane remains against the lower mechanical stops since the restoring force of the linked system piezo actuator/pumping membrane is not sufficient to counterbalance the pressure forces.

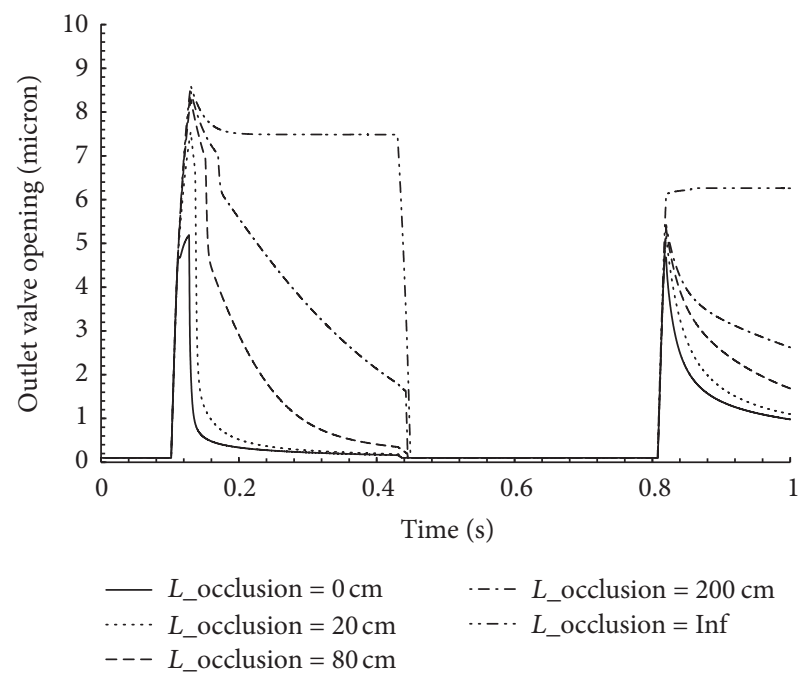

FIGURE 6: Simulated outlet valve opening as a function of the occlusion length defined in (7).

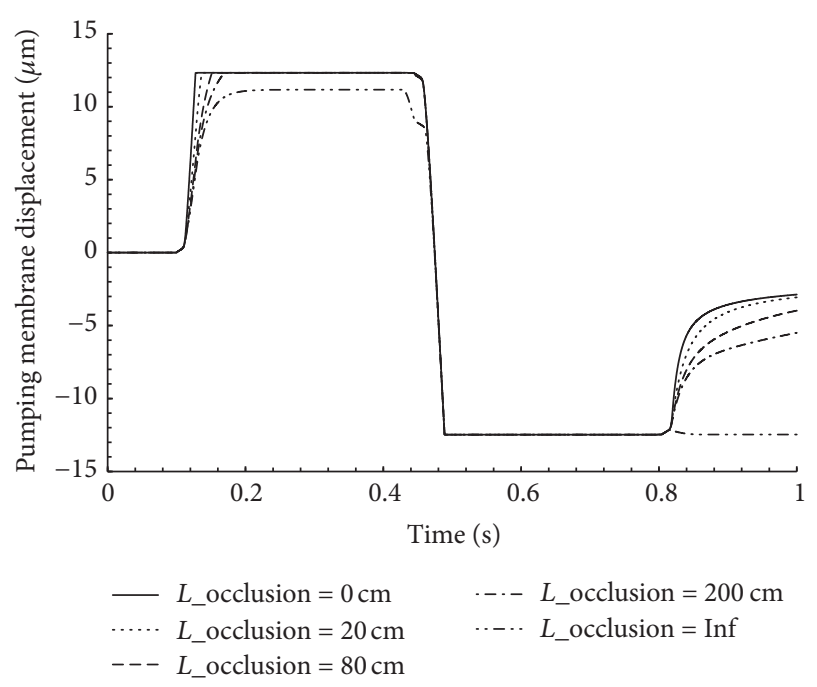

FIGURE 7: Simulated pumping membrane displacement as a function of the occlusion length defined in (7).

\section{Conclusion}

The fluid dynamics in a piezoelectric MEMS micropump dedicated to insulin delivery has been modeled using electrical equivalent networks. The pressures inside the pumping chamber and the cannula have been simulated and compared to experiment to validate the numerical modeling. Partial occlusion has been implemented and tested with success in the simulation tool. The analysis of these simulated pressure profiles during piezo actuation has been notably used to determine the best actuation profile able to infuse with accuracy in presence of partial occlusion. The voltage on the piezo actuator shall be maintained until the pressure in the pumping chamber reaches a threshold value that depends on the valve geometry. This modulation of the actuation cycle is notably dedicated to overnight basal infusion of insulin, 
in order to limit the occurrences of occlusion alarm while maintaining delivery accuracy.

\section{Conflicts of Interest}

The authors declare no conflicts of interest.

\section{Acknowledgments}

The authors would like to thank the whole team involved in the Insulin Micropump Project at Debiotech and ST Microelectronics (MEMS Division, Agrate Brianza, Italy, and Kirkop, Malta). They also explicitly thank Harald van Lintel, Bertrand Boutaud, Véronique Vallet, and Niklaus Schneeberger, who made important contributions to the modeling of this MEMS micropump.

\section{References}

[1] R. Zengerle and M. Richter, "Simulation of microfluid systems," Journal of Micromechanics and Microengineering, vol. 4, no. 4, pp. 192-204, 1994.

[2] T. Bourouina and J.-P. Grandchamp, "Modeling micropumps with electrical equivalent networks," Journal of Micromechanics and Microengineering, vol. 6, no. 4, pp. 398-404, 1996.

[3] T. Bourouina, A. Bossebœuf, and J.-P. Grandchamp, "Design and simulation of an electrostatic micropump for drug-delivery applications," Journal of Micromechanics and Microengineering, vol. 7, no. 3, pp. 186-188, 1997.

[4] Q. Cui, C. Liu, and X. F. Zha, "Simulation and optimization of a piezoelectric micropump for medical applications," International Journal of Advanced Manufacturing Technology, vol. 36, no. 5-6, pp. 516-524, 2008.

[5] I. Izzo, D. Accoto, A. Menciassi, L. Schmitt, and P. Dario, "Modeling and experimental validation of a piezoelectric micropump with novel no-moving-part valves," Sensors and Actuators, A: Physical, vol. 133, no. 1, pp. 128-140, 2007.

[6] S. Tanaka, O. Ichihashi, K. Sugano, T. Tsuchiya, and O. Tabata, "Analysis of valveless piezoelectric micropump using electrical equivalent circuit model," in Proceedings of 4th International Conference on Solid-State Sensors, Actuators and Microsystems, TRANSDUCERS and EUROSENSORS '07, pp. 2183-2186, Lyon, France, June 2007.

[7] Y. C. Hsu and N. B. Le, "Equivalent electrical network for performance characterization of piezoelectric peristaltic micropump," Microfluidics and Nanofluidics, vol. 7, no. 2, pp. 237-248, 2009.

[8] E. Chappel, S. Mefti, G.-L. Lettieri, S. Proennecke, and C. Conan, "High precision innovative micropump for artificial pancreas," in Proceedings of Microfluidics, BioMEMS, and Medical Microsystems XII, San Francisco, Calif, USA, February 2014.

[9] D. Dumont-Fillon, H. Tahriou, C. Conan, and E. Chappel, "Insulin micropump with embedded pressure sensors for failure detection and delivery of accurate monitoring," Micromachines, vol. 5, no. 4, pp. 1161-1172, 2014.

[10] E. Chappel, D. Dumont-Fillon, and S. Mefti, "Passive flow regulators for drug delivery and hydrocephalus treatment," in Proceedings of Microfluidics, BioMEMS, and Medical Microsystems XII, San Francisco, Calif, USA, February 2014.

[11] F. M. White, Viscous Fluid Flow, McGraw-Hill, Boston, Mass, USA, 2nd edition, ISBN: 0-07-069712-4 (1991).
[12] J. Brange, L. Andersen, E. D. Laursen, G. Meyn, and E. Rasmussen, "Toward understanding insulin fibrillation," Journal of Pharmaceutical Sciences, vol. 86, no. 5, pp. 517-525, 1997.

[13] D. Kerr, J. Morton, C. Whately-Smith, J. Everett, and J. P. Begley, "Laboratory-based non-clinical comparison of occlusion rates using three rapid-acting insulin analogs in continuous subcutaneous insulin infusion catheters using low flow rates," Journal of Diabetes Science and Technology, vol. 2, no. 3, pp. 450-455, 2008. 


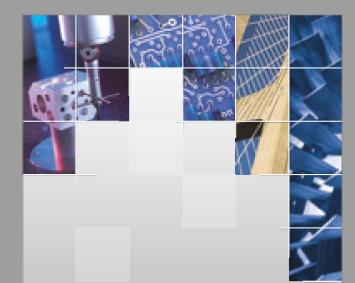

\section{Enfincering}
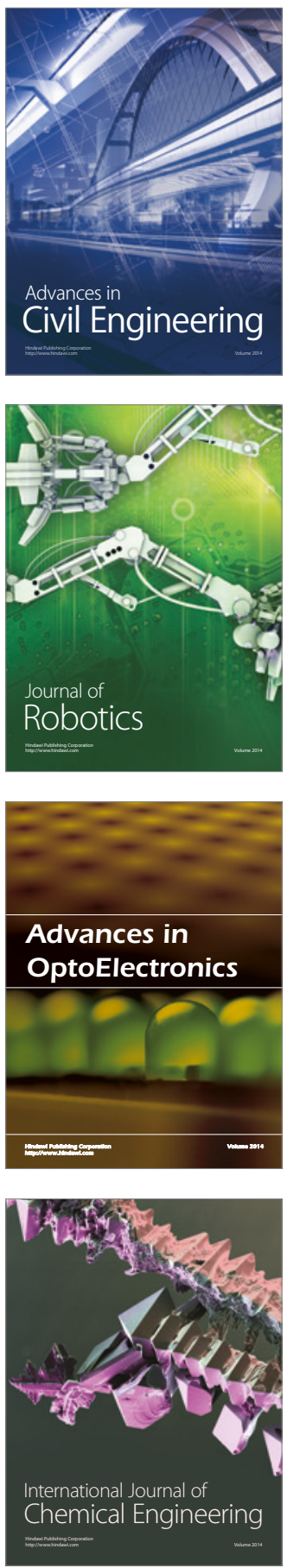

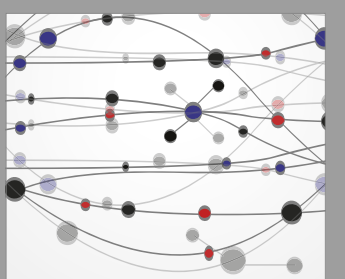

The Scientific World Journal

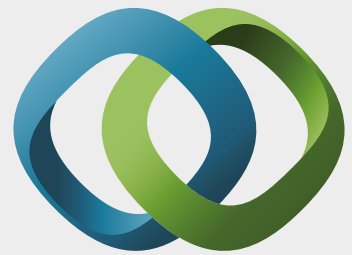

\section{Hindawi}

Submit your manuscripts at

https://www.hindawi.com
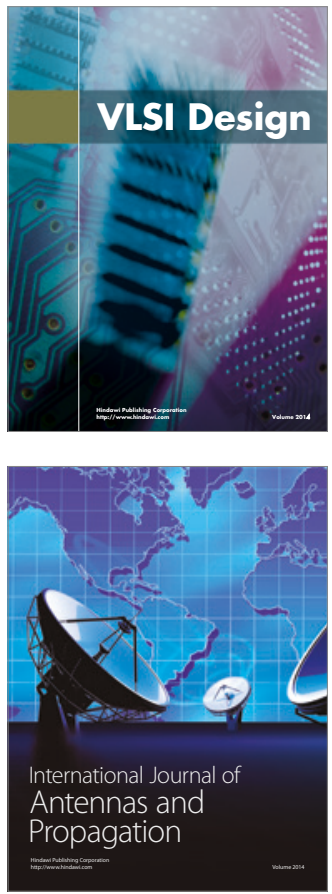

\section{Rotating}

Machinery
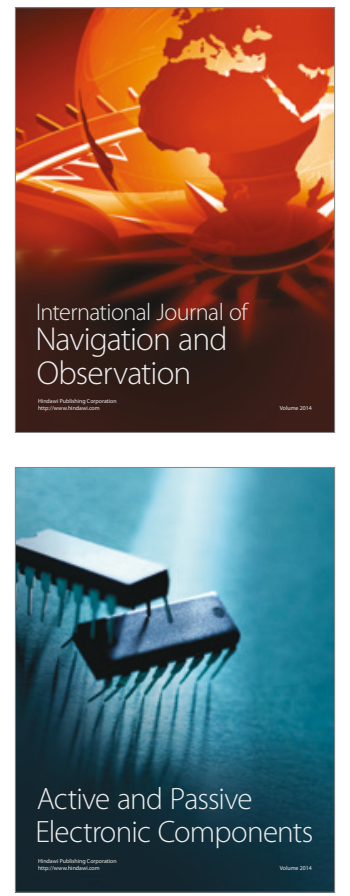
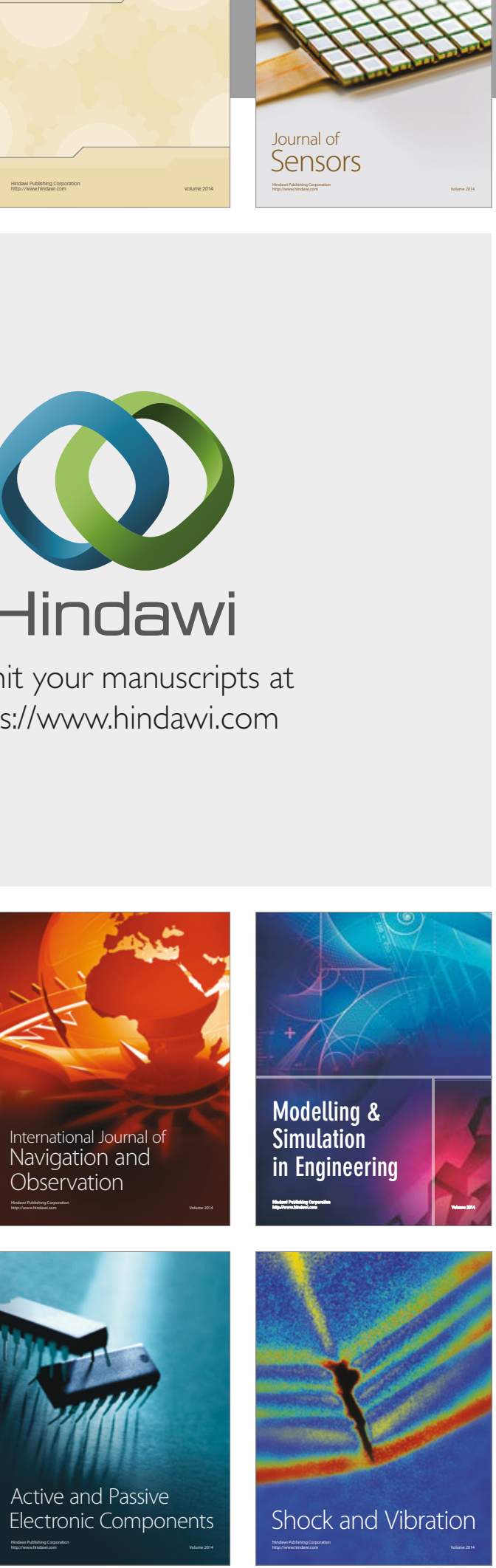
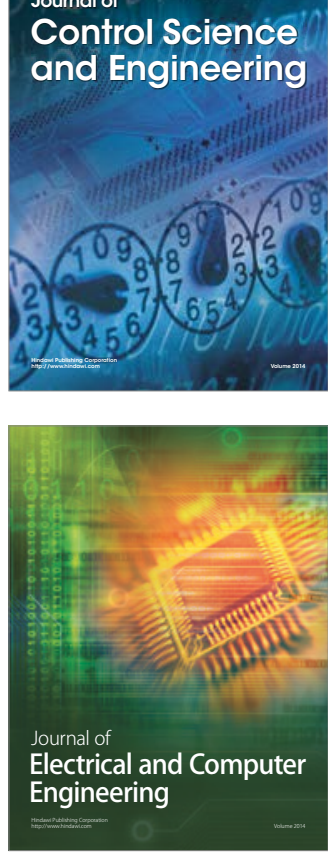

Distributed

Journal of

Control Science

and Engineering
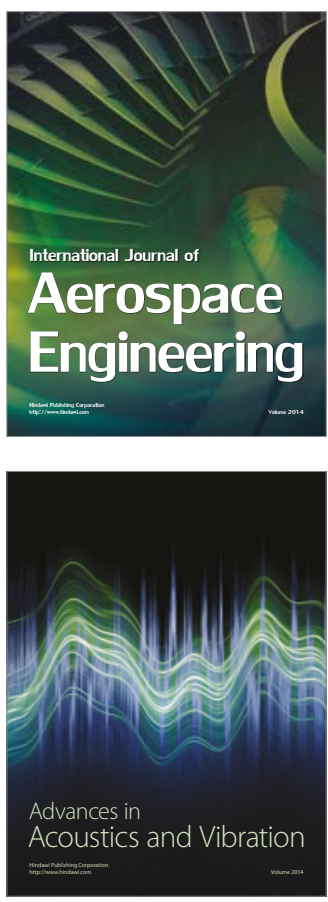

Sensor Networks 